\title{
Moving Effortlessly in Three Dimensions: Does Donders' Law Apply to Arm Movement?
}

\author{
John F. Soechting, ${ }^{2}$ Christopher A. Buneo, ${ }^{1}$ Uta Herrmann, ${ }^{2}$ and Martha Flanders ${ }^{2}$ \\ ${ }^{1}$ Department of Physiology and ${ }^{2}$ Graduate Program in Neuroscience, University of Minnesota, Minneapolis, \\ Minnesota 55455
}

\begin{abstract}
Donders' law, as applied to the arm, predicts that to every location of the hand in space there corresponds a unique posture of the arm as defined by shoulder and elbow angles. This prediction was tested experimentally by asking human subjects to make pointing movements to a select number of target locations starting from a wide range of initial hand locations. The posture of the arm was measured at the start and end of every movement by means of video cameras. It was found that, in general, the posture of the arm at a given hand location does depend on the starting location of the movement and that, consequently, Donders' law is violated in this experimental condition. Kinematic and kinetic factors that could account for the variations in arm posture were investigated. It proved impossible to predict the final posture of the arm purely from kinematics, based on the initial posture of the arm. One hypothesis was successful in predicting final arm postures, namely that the final posture minimizes the amount of work that must be done to transport the arm from the starting location.
\end{abstract}

[Key words: arm movements, Donders' law, minimum work, minimum energy, optimization, reaching]

The search for laws that govern neurally controlled movements is an ongoing one. While many have been proposed, few have withstood the test of time. One of those is Donders' law, first discovered in the middle of the last century. This law states that for every gaze direction, there is a unique orientation of the eyes in the head (cf. Alpern, 1969; Nakayama and Balliet, 1977; Tweed and Vilis, 1990). Stated another way, for each combination of horizontal and vertical deviation of the eye, there corresponds a unique value of ocular torsion. A stronger statement of the law was provided by Listing, who realized that any change in eye position can be achieved by a rotation about some axis in space. Listing's law states that the only possible ocular orientations are those that can be achieved by rotations from a refcrence position (the primary gaze direction), with the axes of these rotations constrained to lie in a plane.

Donders' and Listing's laws hold true independently of previous gaze directions assumed by the eye. For example ocular torsion is the same when the final gaze direction is achieved first

\footnotetext{
Received Mar. 9, 1995; revised May 11, 1995; accepted May 22, 1995

This work was supported by USPHS Grants NS-27484 and NS-15018. We thank two anonymous reviewers for their helpful comments.

Correspondence should be addressed to J. F. Soechting, Department of Physiology, 6-255 Millard Hall, University of Minnesota, Minneapolis, MN 55455. Copyright (C) 1995 Society for Neuroscience $0270-6474 / 95 / 156271-10 \$ 05.00 / 0$
}

by a saccade to the right and then an upward saccade, and when the order of rotations is reversed, the rightward saccade following the upward one. Since rotations do not generally commute (cf. Tweed and Vilis, 1987), Donders' law is not a trivial result of geometric or biomechanical constraints. In fact, it is not obeyed under some conditions, for example during the vestibuloocular reflex (Crawford and Vilis, 1991).

Recently, several groups of investigators have reported that Donders' law is also obeyed during head and arm movements (Straumann et al., 1991; Hore et al., 1992; Miller et al., 1992). There are appreciable differences in the biomechanics of the eye, the head and the arm. For example, the eye can be approximated as a sphere with negligible inertia, with a minimal number of muscles, arranged approximately in orthogonal directions (Robinson, 1982; Simpson and Graf, 1985). The head has considerably more inertia and the arrangement of muscles is much more complex (Keshner et al., 1992). The arm, instead, is a double pendulum and the equations describing its kinematics and kinetics differ substantially from those that describe the motion of a sphere. Thus, the conclusion that the same biological law governs motion of all three systems would have profound implications for neural control mechanisms.

The cited observations on arm movements were made under a restricted set of experimental conditions: pointing at distant targets with an outstretched arm. Thus, it is not known to what extent Donders' law is obeyed by arm movements under more general conditions. It is known that it is not obeyed when subjects arc required to grasp objects (Soechting and Flanders, 1993; Helms Tillery et al., 1995). When grasping a cylindrical object whose tilt is varied, human subjects and nonhuman primates tend to orient their proximal arm (shoulder and elbow) such as to restrict the amount of rotation of the wrist required to align the hand with the cylinder. Since the range of wrist motion is limited, it could be argued that this violation of Donders' law results from biomechanical constraints innposed by the task and that Donders' law might still be applicable for movements that do not require a precise orientation of the hand.

In this article, we describe the results of experiments that were intended to test this possibility. We examined the posture of the arm for pointing movements beginning and ending at a range of targets that spanned the workspace. We find that in general Donders' law is not obeyed: the final posture of the arm depends on the starting location of the hand. The final postures are not arbitrary, however, and we were able to uncover a principle that predicted the arm orientations that we observed. It appears that the movements are organized so as to minimize the amount of energy expended to transport the arm from the initial position to the target. 


\section{Distribution of Hand Locations}

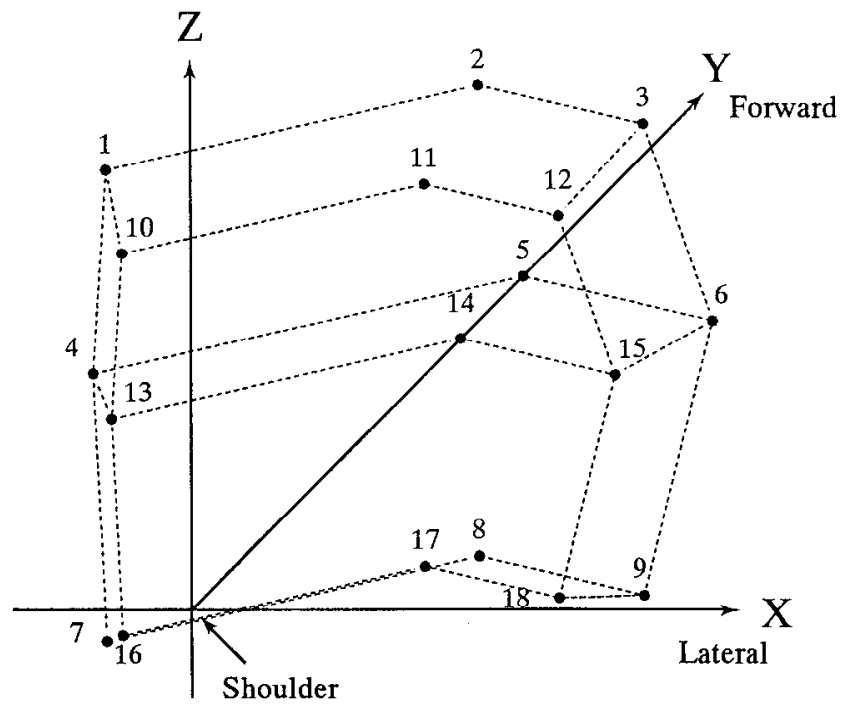

Figure 1. Distribution of starting and final locations of the hand for pointing movements. The points are plotted from the subject's perspective, with the origin of the XYZ coordinate system at the shoulder. Nine of the points are $43 \mathrm{~cm}$ from the shoulder, the others are $53 \mathrm{~cm}$ from the shoulder. The points are located at three values of clevation $\left( \pm 30^{\circ}\right.$ and $0^{\circ}$ ) relative to the horizontal plane passing through the shoulder and in the shoulder's parasagittal plane, $45^{\circ}$ to the left or $30^{\circ}$ to the right.

\section{Materials and Methods}

Experimental design. We examined arm movements beginning at one of 18 locations and ending at one of 5 targets. The distribution of beginning and ending points of the movements is shown in Figure 1. Nine of the positions (numbered 1-9) were at a distance of 53 cin from the subject's shoulder, the other nine (10-18) were more proximal, at a distance of $43 \mathrm{~cm}$. Movements were begun with the hand at shoulder level (4-6 and 13-15), at an elevation of $30^{\circ}$ above the shoulder (1-3 and $10-12$ ) or $30^{\circ}$ below the shoulder (7-9 and 16-18). Six of the points were located in the parasagittal plane passing through the shoulder $(2$, $5,8,11,14,17)$. The others were located either $45^{\circ}$ to the left or $30^{\circ}$ to the right of this plane.

The five targets were located at the extremes of this distribution of points (targets 1, 3, 7, and 9) and in the middle of the work space (target 5 , at a distance of $53 \mathrm{~cm}$ with $0^{\circ}$ elevation and $0^{\circ}$ azimuth). The beginning and ending points of the movements were indicated by means of a pointer grasped in the hand of a robot arm (TeachMover, Microbot Inc.). (The pointer was required to extend the range of locations that could be reached by the robot arm.) The robot positioned the tip of the pointer at one of the starting locations and seated subjects were instructed to touch the tip of the pointer with a pen-shaped stylus grasped in their hand. The subject held his or her arm still while the robot arm was repositioned to one of the five targets. The subjects were then required to touch the pointer's tip at the new location. Starting and ending locations were varied randomly. There were a total of $85 \mathrm{com}-$ binations ( 5 targets $\times 17$ starting locations) and for each subject we obtained either 3 or 5 blocks of 85 trials.

Four subjects participated in these experiments. They were naive as to the purpose of the experiment and were given no instructions other than to move their arm to touch the tip of the pointer. Subjects gave their consent to the experimental procedures.

The posture of the arm was recorded prior to and immediately after the end of each movement by means of two video cameras (VP110, Motion Analysis Corp.). Spherical reflective markers were placed on the right arm of each subject at the shoulder, elbow and wrist. The location of these markers in three-dimensional space was computed off line and shoulder and elbow angles were computed from these values. Motions of the arm and head were not constrained in any way.

Definition of arm posture. Figure 1 also illustrates the coordinate system we used to define target location and arm posture: $\mathrm{X}$ is in the

\section{Arm Orientation}

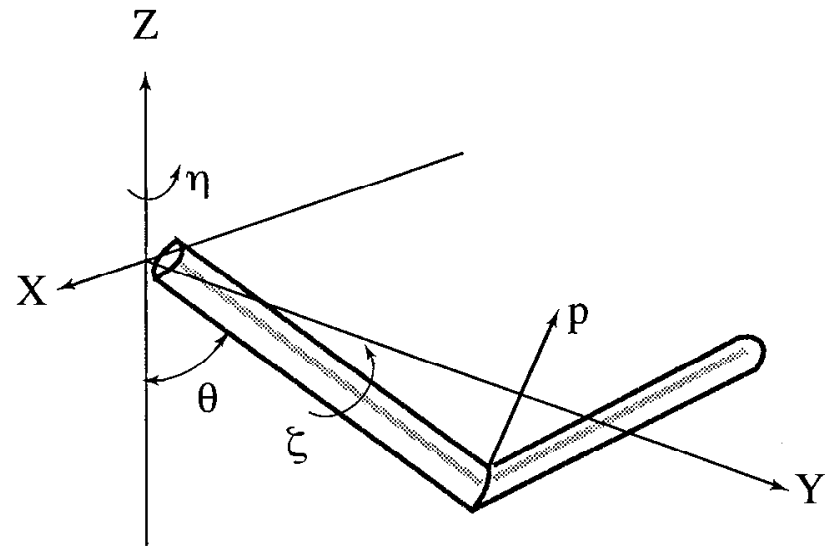

Figure 2. Angles defining the posture of the arm. Three angles are required to define the motion at the shoulder $(\eta, \theta$, and $\zeta)$. The yaw angle $(\eta)$ represents a rotation of the arm about the vertical $Z$ axis, measured relative to the anterior $Y$ direction. The elevation angle $(\theta)$ represents the angle between the arm and the $Z$ axis, measured in the vertical plane. $\theta$ is zero when the upper arm is vertical. The humeral rotation is defined by $\zeta$, $\zeta$ being zero when the plane of the arm is vertical. The perpendicular to this plane (p) provides a succinct description of the arm's posture.

lateral direction, $\mathrm{Y}$ is forward, and $\mathrm{Z}$ is up. The origin of this coordinate system is at the shoulder. Four angles are required to define the posture of the arm in this coordinate system-three resulting from rotations at the shoulder joint and one at the elbow. The angles used to define the rotation at the shoulder are illustrated in Figure 2 (Soechting and Ross, 1984; Soechting et al., 1986). We define arm posture to result from three successive rotations, starting with the upper arm vertical (along the Z-axis) and the arm in the parasagittal (Y-Z) plane passing through the shoulder (if the forearm is not fully extended). The first rotation $(\eta)$ is about the vertical $\mathrm{Z}$-axis and determines the yaw angle of the arm. The second rotation $(\theta)$ is about an axis perpendicular to the plane of the arm (the lateral, $X$-axis if there is zero yaw) and determines the arm's elevation. The third rotation $(\zeta)$ is about the humeral axis. This rotation does not change the location of the elbow but does affect the location of the wrist in space and the plane of the arm. We also define $\phi$ to be the angle of flexion of the forearm, $\phi=0$ corresponding to full extension. by

With these definitions, the location of the elbow $\left(x_{c}, y_{c}, z_{c}\right)$ is given

$$
\begin{aligned}
& x_{e}=-l_{a} \sin \eta \sin \theta \\
& y_{e}=l_{a} \cos \eta \sin \theta \\
& z_{e}=-l_{a} \cos \theta
\end{aligned}
$$

where $l_{a}$ is the length of the upper arm. The location of the wrist $\left\{x_{w}, y_{w}, z_{w}\right\}$ is given by

$$
\begin{gathered}
x_{w}=x_{e}-l_{j}[\sin \phi(\cos \zeta \sin \eta \cos \theta+\sin \zeta \cos \eta) \\
\quad+\cos \phi \sin \eta \sin \theta] \\
\begin{aligned}
y_{w}=y_{e}+l_{j}[\sin \phi(\cos \zeta \cos \eta \cos \theta-\sin \zeta \sin \eta) \\
\quad+\cos \phi \cos \eta \sin \theta]
\end{aligned} \\
z_{w}=z_{e}+l_{f}[\sin \phi \cos \zeta \sin \theta-\cos \phi \cos \theta],
\end{gathered}
$$

where $l_{f}$ is the length of the forearm.

The yaw angle $(\eta)$ and the upper arm elevation $(\theta)$ were computed from the measured location of the elbow relative to the shoulder. The angle of forearm flexion $(\phi)$ was computed as the angle between the vector connecting the elbow to the shoulder and the vector from the elbow to the wrist. To determine the angle of humeral rotation $(\zeta)$, we first computed the normal p to the plane of the arm (Fig. 2) from the cross product of the vector connecting the elbow to the shoulder with 

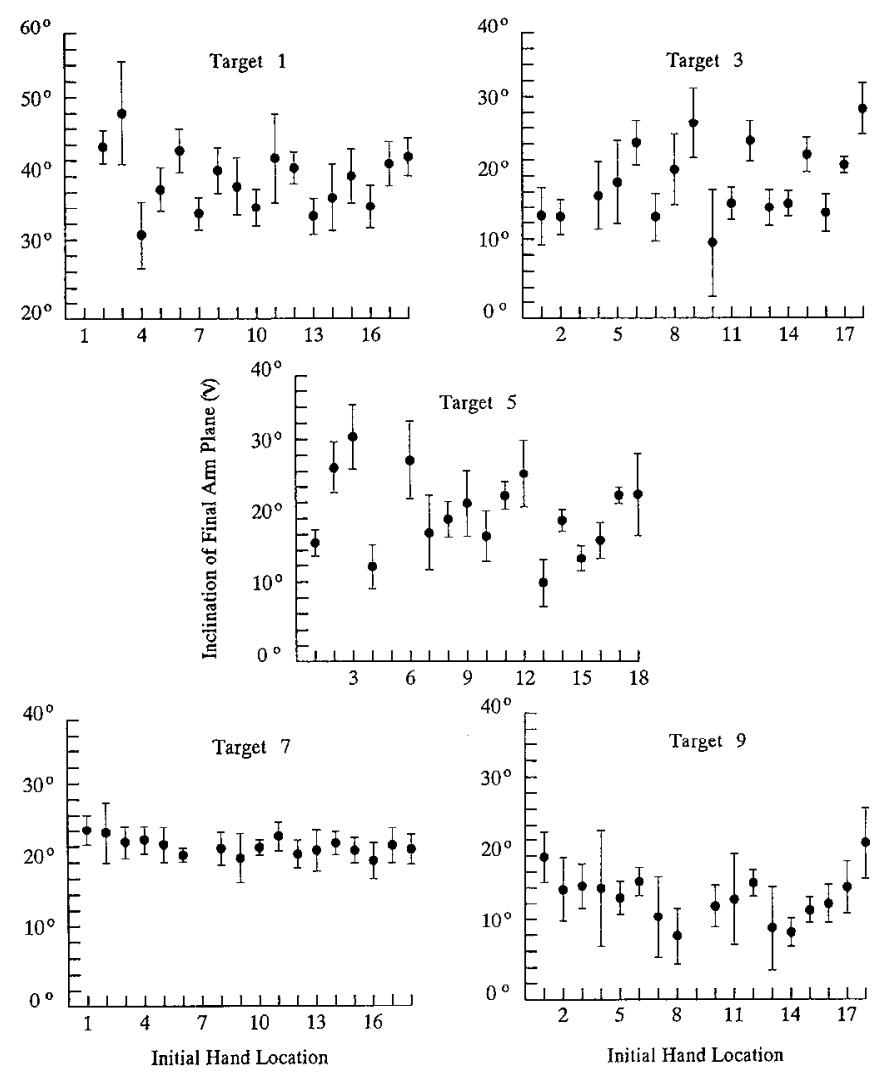

Figure 3. The variation in the inclination of the plane of the arm with the hand at each of the five targets. The inclination $(v)$ is the angle between the perpendicular $p$ to the plane of the arm and the horizontal plane. For each target, values ( \pm 1 SD) are shown for each of the 17 starting locations. The data are from Subject 1.

the vector from the elbow to the wrist. We define $v$ to be the angle $\mathbf{p}$ makes with the horizontal plane. The angle $v$ is related to the humeral rotation $(\zeta)$ by

$$
\sin v=\sin \theta \sin \zeta .
$$

As shown in Figure 2 by the direction of the arrows, internal humeral rotation is defined to be positive, as is yaw directed medially from the parasagittal plane through the shoulder.

The parameter $v$ provides the most economical way to describe variations in arm posture for a given hand location-once $v$ is determined, the shoulder angles $(\eta, \theta$, and $\zeta)$ are determined uniquely. The elbow angle $\phi$ depends only on the distance of the target from the shoulder and thus, it is determined uniquely by hand location.

In order to facilitate a comparison of our results with those obtained by other investigators (Straumann et al., 1991; Hore et al., 1992; Miller et al., 1992) we also computed the quaternion vector that describes rotation at the shoulder joint (Westheimer, 1957). The quaternion vector defines the rotation about a single axis that takes the arm from a reference posture to its present posture. We followed the procedures adopted by Hore et al. (1992). In the reference position, the upper arm is horizontal, parallel to the $\mathrm{Y}$-axis, and the plane of the arm is vertical. We computed the components of this rotation vector along the three Cartesian axes: V, a rotation about the vertical $\mathrm{Z}$-axis; $\mathrm{H}$, a rotation about the horizontal $\mathrm{X}$-axis; and $\mathrm{I}$; a torsional rotation about the $\mathrm{Y}$-axis.

\section{Results}

Arm posture at the target depends on the initial posture. Donders' law does not hold for arm movements. In all subjects, the posture of the arm at the target location depended significantly (ANOVA, $F$ test, $p<0.01$ on the inclination $v$ ) on the starting location of the hand for at least some of the targets. Results from two of the subjects are illustrated in Figures 3 and 4.
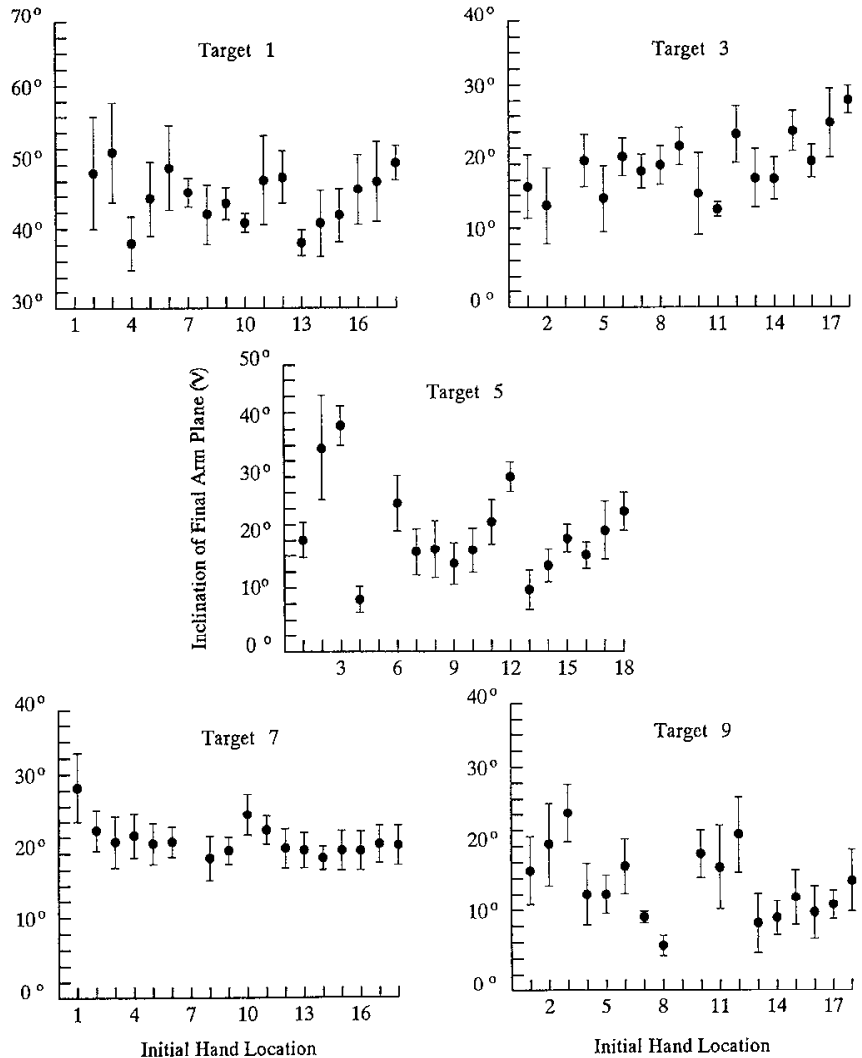

Figure 4. Dependence of final arm posture on initial location of the hand. Data are from Subject 2 and are plotted in the same format as those in Figure 3.

The plots in Figures 3 and 4 show the mean ( \pm 1 SD) of the inclination ( $v$ ) of the normal $\mathbf{p}$ to the plane of the arm for each of the targets and each of the starting locations. The greatest amount of postural variation was observed for the middle target (5) located at shoulder level in the mid-sagittal plane. For this target location, $v$ depended on the starting location of the hand for all four subjects. The least amount of postural variation was observed for target location 7 (lower left). In fact, for this target, the final arm posture did not depend significantly on the starting arm posture for the subject whose results are illustrated in Figure 3. For target $7, v$ did depend on initial hand location for the results illustrated in Figure 4, as well as in one of the two other subjects. In particular, in Figure 4 , the arm posture at target 7 for movements beginning from target 1 differed significantly ( $p$ $<0.01$, post hoc $t$ test with Bonferroni adjustment) from the postures for movements starting from many of the other locations (numbers 5, 8, 9, 12-18). None of the other arm postures differed significantly from each other in this example, which illustrates the minimal amount of postural variation. By contrast, for target 5, pairwise comparisons showed each of the final postures differed significantly $(p<0.01)$ from at least one other final posture.

The dependence of the inclination of the arm's plane on the starting location of the hand was not idiosyncratic for each subject. We have already mentioned that in all subjects the values varied least for target 7 and varied most for target 5. An examination of the variations in the pattern for each target location demonstrates additional consistencies. For example, at target 5 , the final inclination was least for initial positions 4 and 13 for 


\begin{tabular}{|c|c|c|c|c|c|c|}
\hline Target & $\eta$ & $\theta$ & $\zeta$ & V & $\mathbf{H}$ & $\mathrm{T}$ \\
\hline \multirow[t]{4}{*}{1} & $-1.1(6.1)$ & $67.8(3.7)$ & $43.4(5.1)$ & $7.1(5.8)$ & $21.1(2.3)$ & $-41.4(4.0)$ \\
\hline & $-0.5(6.9)$ & $65.8(3.9)$ & $51.6(4.9)$ & $9.9(6.5)$ & $22.1(2.6)$ & $-48.7(3.6)$ \\
\hline & $7.8(4.2)$ & $75.7(4.9)$ & $43.2(4.3)$ & $12.3(5.0)$ & $10.5(3.8)$ & $-42.7(3.5)$ \\
\hline & $-13.7(6.2)$ & $62.6(4.5)$ & $47.3(4.7)$ & $-1.4(6.0)$ & $30.2(2.8)$ & $-41.4(3.6)$ \\
\hline \multirow[t]{4}{*}{3} & $-44.7(5.9)$ & $62.2(3.1)$ & $21.6(6.8)$ & $-36.7(4.0)$ & $33.2(2.3)$ & $-8.9(5.4)$ \\
\hline & $-52.4(8.1)$ & $55.3(2.9)$ & $24.4(5.9)$ & $-40.6(5.8)$ & $40.4(1.8)$ & $-6.0(4.0)$ \\
\hline & $-45.5(5.8)$ & $68.3(4.4)$ & $19.8(5.4)$ & $-39.4(4.6)$ & $27.2(2.9)$ & $-9.6(5.2)$ \\
\hline & $-63.9(8.3)$ & $64.1(3.4)$ & $39.7(5.0)$ & $-48.0(7.2)$ & $40.4(3.0)$ & $-19.3(5.4)$ \\
\hline \multirow[t]{4}{*}{5} & $-22.9(6.9)$ & $41.6(3.2)$ & $32.0(8.7)$ & $-7.4(3.3)$ & $50.2(1.8)$ & $-19.3(5.6)$ \\
\hline & $-29.3(10.3)$ & $34.9(5.6)$ & $38.3(12.2)$ & $-7.6(5.0)$ & $57.4(2.4)$ & $-19.7(7.2)$ \\
\hline & $-20.6(5.8)$ & $46.8(5.4)$ & $31.9(8.2)$ & $-7.0(2.9)$ & $45.3(4.1)$ & $-21.6(6.2)$ \\
\hline & $-40.4(4.6)$ & $42.1(3.2)$ & $46.6(6.2)$ & $-16.0(2.5)$ & $54.5(2.0)$ & $-24.0(4.6)$ \\
\hline \multirow[t]{4}{*}{7} & $-10.5(4.2)$ & $24.9(2.0)$ & $64.3(5.3)$ & $25.2(1.3)$ & $56.7(2.1)$ & $-46.3(3.0)$ \\
\hline & $-13.1(7.6)$ & $22.6(3.2)$ & $77.0(7.1)$ & $30.9(2.5)$ & $56.3(3.1)$ & $-53.2(3.4)$ \\
\hline & $-7.7(2.4)$ & $35.8(2.5)$ & $75.5(2.7)$ & $26.4(1.5)$ & $45.3(2.4)$ & $-59.4(2.1)$ \\
\hline & $-38.4(8.9)$ & $21.1(2.2)$ & $84.5(4.6)$ & $18.1(5.8)$ & $66.0(3.1)$ & $-44.0(5.7)$ \\
\hline \multirow[t]{4}{*}{9} & $-64.1(12.4)$ & $18.6(3.9)$ & $50.3(13.0)$ & $-20.5(5.3)$ & $72.4(2.0)$ & $-1.4(7.4)$ \\
\hline & $-107.1(13.0)$ & $14.9(5.9)$ & $81.3(7.4)$ & $-28.2(9.3)$ & $78.0(3.7)$ & $7.3(13.8)$ \\
\hline & $-83.5(14.4)$ & $17.8(4.2)$ & $65.6(15.0)$ & $-24.2(2.7)$ & $76.1(2.7)$ & $0.6(5.0)$ \\
\hline & $-83.3(5.8)$ & $25.9(3.8)$ & $69.4(7.6)$ & $-27.2(2.0)$ & $74.1(1.6)$ & $-8.0(5.4)$ \\
\hline
\end{tabular}

Mean values ( \pm 1 SD) for arm orientation for each target and each subject. Arm orientation is defined in terms of the orientation angles illustrated in Figure 2 and the angular rotation about horizontal (H), vertical (V), and torsional (T) axes from the primary position of the arm.

Subject 1 (Fig. 3) as well as for Subject 2 (Fig. 4). It was greatest for position 3. A third subject also showed a very similar pattern for target 5. (The results for the fourth subject did not conform as well to the general trend.) An examination of the results for the other targets reveals other similarities. For example, when the movements were to target $1, v$ was generally least for starting position 4 and greatest for position 3 .

To determine the extent to which all subjects showed a similar pattern of behavior, we computed correlation coefficients for $v$ between each of the possible pairs of subjects for each of the 5 target locations. Except for target 7, there was a significant $(p$ $<0.01, \chi^{2}$ statistic), overall positive correlation between subjects in the planar inclination of the arm. The patterns for the first three subjects generally showed significant correlations $(p<$ 0.01 ), whereas the behavior of the fourth subject was generally uncorrelated with the behavior of the first three.

The results from all four subjects are summarized in Table 1, where we present the average ( $\pm 1 \mathrm{SD}$ ) for each target. Arm posture is defined in two ways: by three successive rotations ( $\eta$, $\theta$, and $\zeta$; Fig. 2) and by the components of the quaternion vector $(\mathrm{V}, \mathrm{H}$, and $\mathrm{T})$. (There is an approximate correspondence between the two descriptions: $\eta$ and $\mathrm{V}, \theta$ and $\mathrm{H}$, and $\zeta$ and T. Changes in $\theta$ and $\mathrm{H}$ are negatively correlated, because $\theta$ is defined as a rotation starting with the upper arm vertical and $\mathrm{H}$ as a rotation starting with the upper arm horizontal. The correspondence is not exact because $\eta, \theta$, and $\zeta$ are defined as three successive rotations about axes fixed to the arm, whereas $\mathrm{V}, \mathrm{H}$, and $\mathrm{T}$ are the components (along fixed axes) of a single rotation.) In contrast to results of previous studies, torsion $(\mathrm{T})$ varied considerably from target location to target location, spanning $50^{\circ}$. The SD in T was comparable to the SDs of the other angular measures (average value of $5.2^{\circ}$ ).

What factors determine the final posture of the arm? In the previous section, we showed that the posture of the arm de- pended on the starting location of the movement. We also showed that the pattern of this dependence was generally consistent from subject to subject, suggesting that one should be able to uncover an explanation for this phenomenon at a more fundamental level.

We began by asking whether or not kinematic factors could account for the data. By kinematic factors, we mean possibilities such as: does the final posture of the arm minimize the amount of excursion in one or more of the joint angles of the arm? Figure 5 presents a graphical depiction of the results of this investigation. The illustration is for Subject 1, target 5. Any given arm posture is depicted as a point in the three dimensional space of shoulder angles $(\eta, \theta$, and $\zeta$ ). If the hand remains at a fixed location, the angles at the shoulder $(\eta, \theta$, and $\zeta$ ) covary as the inclination of the plane of the arm (v) is changed such that not all points in this space are possible combinations.

As mentioned in Materials and Methods, the elbow angle $(\phi)$ is determined solely by the distance of the target from the shoulder. Therefore, $\phi$ will not vary with $v$. For the sake of argument, assume that the arm is in the vertical plane with the hand at the target $(v=0)$. Keeping the hand on the target and changing the plane of the arm slowly ( $v$ increasing), so that the normal $\mathbf{p}$ points upward (Fig. 2), will cause the elbow to be translated upwards and laterally. Accordingly, the elevation $(\theta)$ will increase, and the yaw angle $(\eta)$ will decrease. The humeral rotation ( $\zeta$ ) will also increase as the inclination of the plane of the arm (v) is increased (Eq. 3). This pattern of covariation is depicted in Figure 5 by the heavy line arising out of the $(\eta-\theta)$ plane. The shading indicates the projection of the $\eta-\theta-\zeta$ curve onto the plane.

The lighter, straight solid lines in the figure connect the initial postures of the arm for each of the 17 starting locations with the final posture at target 5. (The dashed lines represent the projections onto the $\eta-\theta$ plane.) As can be seen from this figure, 


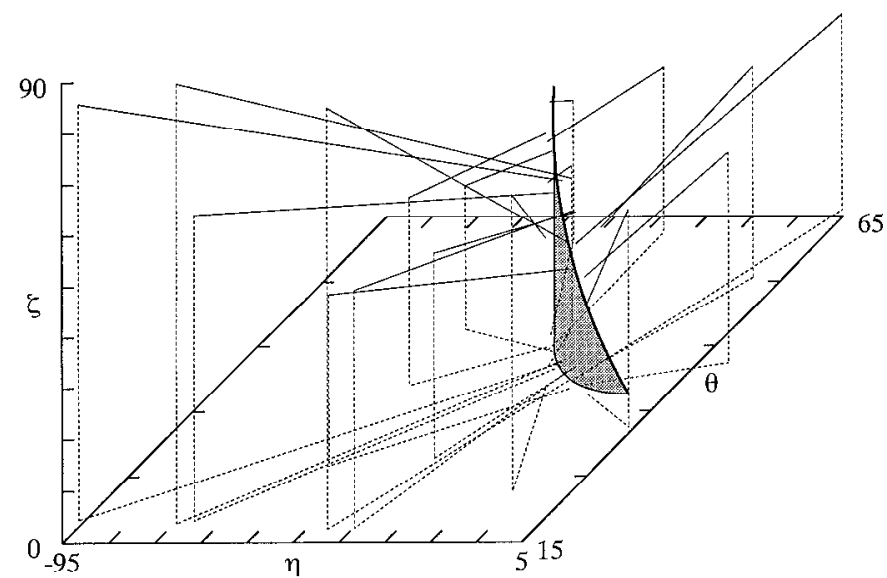

Figure 5. Distribution of arm trajectories in joint space. The straight, solid lines connect the initial and final postures of the arm for movements to target 5 by Subject 1 . The data are plotted in a three-dimensional plot in the space of shoulder angles (yaw, $\eta$; elevation, $\theta$; and humeral rotation, $\zeta$ ). The dashed lines show the projection of these trajectories on the $\eta-\theta$ plane. The heavy curved line shows the computed covariation of the three angles with the hand at the target. Note that the final arm postures are widely dispersed and that there is no consistent kinematic relation between starting and final arm posture. The solid lines do not terminate exactly on the curve because subjects did not always move their hands precisely to the target and because of the extra degrees of freedom afforded by the wrist and fingers. However, the distance between the ends of the solid lines and the curve for each posture is much smaller than is the dispersion of the various postures along the curve, suggesting that these sources of variability are minor compared to the postural changes of the proximal arm.

the starting locations spanned a wide range of arm postures and the final arm postures at target 5 are widely dispersed along the curve of permissible values.

There is no obvious pattern that emerges from an inspection of Figure 5. For example, it does not appear that the final posture of the arm minimizes the amount of excursion in one or all of the joint angles. If the total angular excursion were minimized, the straight lines in Figure 5 would be the shortest possible and each of the linear trajectories would be perpendicular to the line of permissible values. This is clearly not the case. Nor do the final shoulder angles appear to be correlated with the initial values in Figure 5. This was tested quantitatively. We were unable to discover any strong correlations between the initial and final elevation and yaw angles. We were also unable to find any strong correlations between the plane of the arm at the onset of the movement and at its termination.

This latter finding is illustrated for the first two subjects in Figure 6 (see also Figs. 3 and 4). In this figure we show schematically the inclination of the arm's plane for the movements to target 5 beginning at other distal locations $(1-4,6-9)$. The starting inclination is indicated by the lightly shaded lines, the inclination at target 5 by the solid line. This schematic again demonstrates the consistency in the behavior of the two subjects. The largest changes in the arm's inclination were for movements beginning at locations 1 and 3. For initial location 3, the plane of the arm rotated in a counterclockwise direction (increasing $v$ ), whereas for initial location 1, the plane of the arm was rotated in a clockwise direction. These two particular results suggested an answer to the question: what can account for the manner in which the plane of the arm varies?

Consider a movement from location 3 to location 5 (Fig. 7). If the arm is in a plane that is close to vertical at location 3 ,

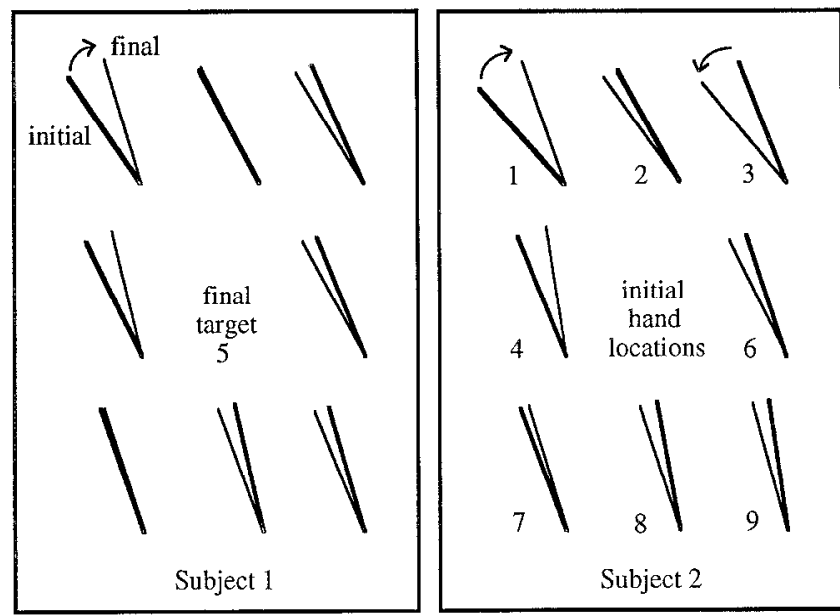

Figure 6. Relations between initial and final plane of the arm for movements from the eight distal locations to target 5 . Data are for the same two subjects as those in Figures 3 and 4 . The initial plane of the arm is denoted by light shading, the final plane by the solid line. Note that the plane of the arm is rotated in a clockwise direction in moving from 1 to 5 , and in a counterclockwise direction when the arm is moved from 3 to 5 .

then location 5 can be reached approximately by a pure rotation about the axis of the humerus. This is illustrated schematically in Figure 7 , by rotation 1 . The target could also be reached by a variety of other rotations. In fact, any rotation that is a combination of rotation 1 plus a rotation about the axis from the shoulder to the hand is permissible. One such example is indicated schematically as a rotation about axis 2 in Figure 7 . Now the moment of inertia of the arm is much less for humeral rotations (about the long axis of the arm), than it is for rotations about any other axis. This implies that it requires much less work to rotate the upper arm about the humeral axis than about any

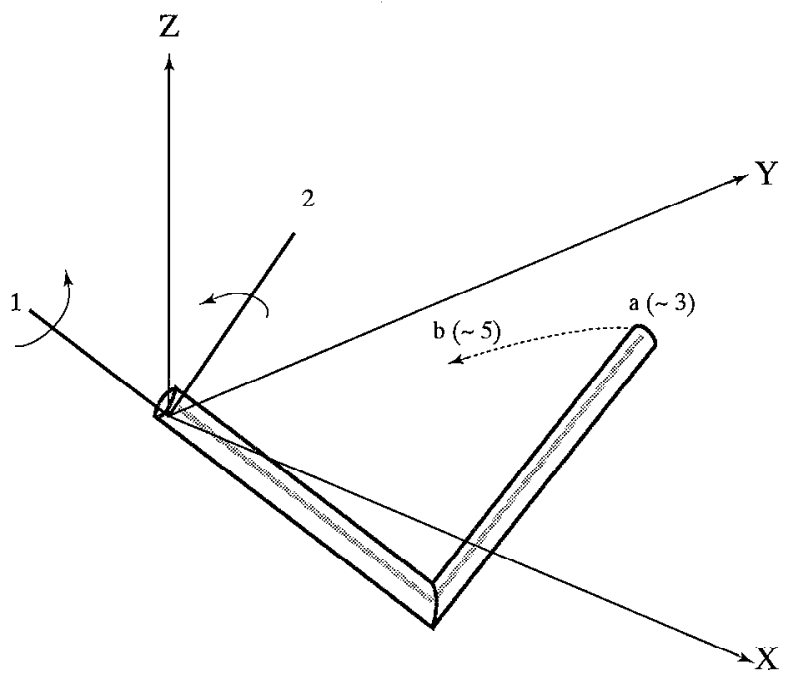

Figure 7. Schematic of possible arm movements from a (approximating location 3) to $b$ (approximating target 5). Given the location of these two points, the movement can be accomplished primarily by a rotation about the axis of the humerus. Other possible solutions are the sum of this rotation about axis 1 plus a rotation of arbitrary size about the axis connecting shoulder and hand (at $a$ ). One other possible solution is a rotation around axis 2 . Note that the inertia of the arm along the humeral axis is much less than the inertia of the arm about any other axis. Consequently, rotations about axis 1 would require much less work. 
other axis. A similar argument also holds for movements to target 5 from location 1 (cf. Fig. 6). In this case, energy considerations would predict a humeral rotation in the clockwise direction, in agreement with experimental observations. Since the hypothesis appeared plausible, we then set out to test it quantitatively.

In actuality, things are a bit more complicated. First, one needs to consider also the amount of work required to rotate the forearm; this would be minimum for rotations about the long axis of the forearm. Secondly, target 5 (for example) cannot in fact be reached from location 3 solely by a pure humeral rotation. To test this hypothesis more precisely, we computed the amount of work required to move the arm.

The principle of minimum work. The amount of work $W$ that is required to move the arm from point a to point $b$ is given by

$$
W=\int \mathbf{T} \cdot d \Theta
$$

where $\boldsymbol{T}$ is the vector of torques at the shoulder and elbow and $\theta$ is the vector of angular displacements at the shoulder and elbow $\{\eta, \theta, \zeta, \phi\}$. Equation 4 may be rewritten as

$$
W=\int \mathbf{T} \cdot \Omega d t=1 / 2 m v^{2}+1 / 2 \Omega^{T} \cdot I \Omega
$$

where $\Omega=d \theta / d t$ and $m$ is the mass of the arm, $v$ the speed of the arm's center of mass, and $I$ is the inertia tensor of the arm. We define a coordinate system $(x, y, z)$ aligned with the arm at every instant in time and coincident with the $(x, y, z)$ coordinate system fixed in space when the upper arm is vertical and in the parasagittal plane. In this coordinate system, the angular velocity of the upper arm is

$$
\begin{aligned}
& \Omega_{x}=\dot{\eta} \sin \zeta \sin \theta+\dot{\theta} \cos \zeta \\
& \Omega_{y}=\dot{\eta} \cos \zeta \sin \theta-\dot{\theta} \sin \zeta \\
& \Omega_{z}=\dot{\eta} \cos \theta+\dot{\zeta} .
\end{aligned}
$$

The $y$ and $z$ components of the angular velocity of the forearm are the same, and its $x$ component is $\Omega_{x f}=\Omega_{x}+\phi$. Assuming the upper arm has a cylindrical shape, its moments of inertia, computed about the center of mass, are the same for the $x$ and $y$ components $\left(I_{a 1}\right)$ and the $z$ component $\left(I_{a 2}\right)$ is much smaller. The same holds true for the forearm: by rotational symmetry, the moment of inertia of the forearm is the same $\left(I_{f 1}\right)$ about any axis perpendicular to the long axis of the forearm (along the radius and ulna). The speed of the arm's center of mass can be computed from the vector cross product between the angular velocity $\Omega$ and the distance $r$ from the shoulder to the arm's center of mass: $\mathbf{v}=\Omega \times \mathbf{r}$. After some algebra, one obtains, for the work $W$,

$$
\begin{aligned}
W=1 / 2[ & I_{1}\left(\dot{\eta}^{2} \sin ^{2} \theta+\dot{\theta}^{2}\right)+I_{2}(\dot{\eta} \cos \theta+\dot{\zeta})^{2} \\
& +I_{3}\left(\Omega_{x}^{2}+\Omega_{y}^{2} \cos ^{2} \phi+\Omega_{z}^{2} \sin ^{2} \phi+\dot{\phi}^{2}+2 \dot{\phi} \Omega_{x}\right. \\
& \left.\quad+2 \Omega_{z} \Omega_{y} \cos \phi \sin \phi\right) \\
& +I_{4}\left(\Omega_{y}^{2} \sin ^{2} \phi+\Omega_{z}^{2} \cos ^{2} \phi-2 \Omega_{z} \Omega_{y} \cos \phi \sin \phi\right) \\
& \left.+A\left(\Omega_{y}^{2} \cos \phi+\Omega_{x}^{2} \cos \phi+\Omega_{z} \Omega_{y} \sin \phi\right)\right],
\end{aligned}
$$

where

$$
\begin{aligned}
& I_{1}=I_{a 1}+m_{a} a_{a}^{2}+m_{f} l_{a}^{2} \\
& I_{2}=I_{a 2} \\
& I_{3}=I_{f 1}+m_{f} a_{f}^{2} \\
& I_{4}=I_{f 2} \\
& A=m_{f} l_{a} a_{f}
\end{aligned}
$$

and $l_{a}$ and $l_{f}$ are the lengths of the upper arm and forearm, $a_{a}$ and $a_{f}$ the distance (from the shoulder or the elbow) to the center of mass of the arm and forearm, and $m_{a}$ and $m_{f}$ are the masses of the two limb segments.

Typical values for these parameters (Soechting et al., 1986), used in the computations to be described below are $I_{1}=0.312$ $\mathrm{kg}-\mathrm{m}^{2}, I_{2}=0.003 \mathrm{~kg}-\mathrm{m}^{2}, I_{3}=0.123 \mathrm{~kg}-\mathrm{m}^{2}, I_{4}=0.0026 \mathrm{~kg}-\mathrm{m}^{2}$, and $A=0.181 \mathrm{~kg}-\mathrm{m}^{2}$.

We examined the following hypothesis: the posture of the arm at the end of the movement is such that the peak work $W$, given by Equation 7 is minimized subject to the constraint that the arm reach the target, that is, Equation 2.

Simplifying assumptions. The total work done during the movement from the starting location to the target is zero; the positive work done to accelerate the arm initially is canceled by the negative work required to decelerate the arm at the end of the movement. The work will assume a peak positive value when the torque changes sign from positive to negative, that is, at the peak of the velocity. Our hypothesis, stated more precise$1 y$, is that the quantity that is minimized is the peak value of work. To compute this precisely, one would need to define the trajectory of the arm from the initial posture to the final posture. To simplify the analysis, we made two assumptions which we will justify in the Discussion: (1) all of the angular velocities reach their peak value at the same time, and (2) the peak angular velocity is proportional to the amount of change in that particular angle. For example, $\dot{\theta} \approx \theta_{t}-\theta_{s}$, where the subscripts $t$ and $s$ refer to the value of $\theta$ at the target and at the starting location. The initial values of $(\eta, \theta, \zeta, \phi)$ were measured, as was the final value of $\phi$, and the final values of $(\eta, \theta, \zeta)$ were chosen so as to minimize Equation 7 subject to the constraint given by Equation 2. This was achieved using the simplex method developed by Nelder and Mead (1964) as described in Press et al. (1992).

Final arm posture can be predicted from minimum work. Figure 8 summarizes the results of the predictions of final arm posture based on minimizing the work done to propel the arm (Eq. 7). For each of the four subjects, we show the correspondence between the amount of humeral rotation $(\zeta)$ predicted by Equation 7 and the amount measured at the end of the movements. Data for each of the five target locations are indicated by different symbols. Overall, there was a high degree of correlation between the predicted and experimental values, with correlation coefficients ( $r^{2}$ values) ranging from 0.891 to 0.925 (Table 2). Furthermore, in all four subjects, the slope of the relationship between measured and predicted values is close to $45^{\circ}$. Our hypothesis was also able to predict the inclination $v$ of the plane of the arm (Figs. 3,4). The correlation coefficients between predicted and experimental values for $v$ are listed in Table 3 . The overall $r^{2}$ values for $v$ are somewhat smaller than those for humeral rotation $\zeta$, ranging from 0.620 to 0.774 .

Generally, there was also a highly significant correlation between measured and predicted values of humeral rotation $\zeta$ and planar inclination $v$ when the data for each of the targets were 

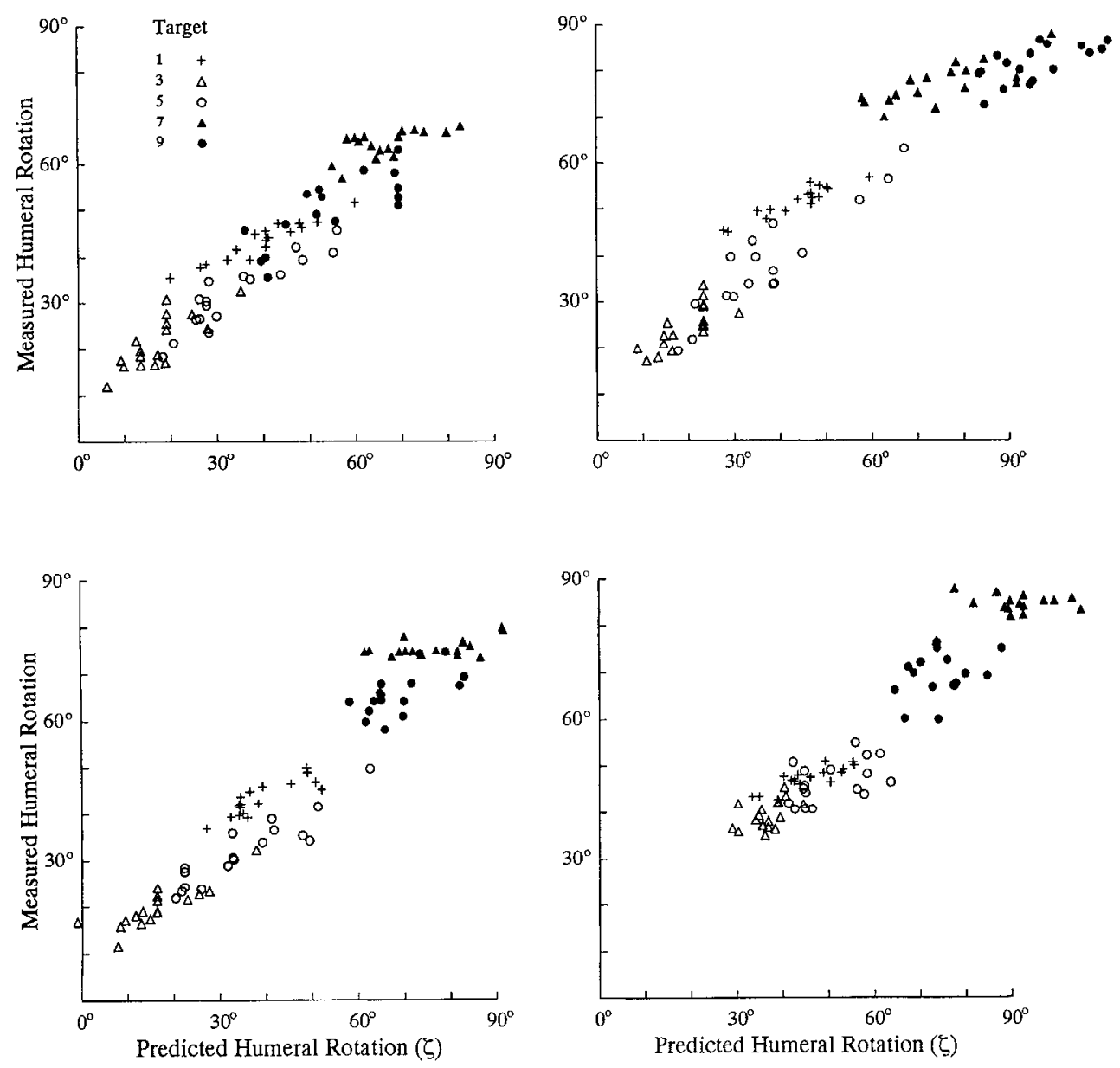

Figure 8. Measured humeral rotation plotted as a function of the humeral rotation predicted by assuming the final arm posture minimizes the amount of energy required to move the arm. Data for all four subjects are shown, subjects 1 and 2 in the top row, 3 and 4 in the bottom row. The different symbols denote the five different target locations.

examined individually (Tables 2, 3). The extent of this correlation differed for different targets, generally being least for targets 7 and 9. For these targets (especially for target 7), the slope of the relation between measured and predicted values of $\zeta$ was considerably less than unity, the actual data showing much less variability than predicted (see also Figs. 3 and 4).

Figure 9 repeats the plot illustrated in Figure 5, this time using predicted (rather than actual) data for target 5 and Subject 1 . The pattern of the relationship between initial and final postures (or more precisely, the lack of a pattern) is similar to that exhibitcd by the actual data in Figure 5 .

Thus, the results shown in Figures 8 and 9 show that the hypothesis that peak work is being minimized by the CNS during arm movements is quantitatively consistent with the data.

\begin{tabular}{|c|c|c|c|c|}
\hline & Subject 1 & Subject 2 & Subject 3 & Subject 4 \\
\hline Overall & 0.891 & 0.914 & 0.925 & 0.905 \\
\hline Target 1 & 0.907 & 0.878 & 0.722 & 0.710 \\
\hline Target 3 & 0.625 & 0.563 & 0.727 & 0.253 \\
\hline Target 5 & 0.846 & 0.848 & 0.848 & 0.235 \\
\hline Target 7 & 0.430 & 0.574 & 0.234 & 0.057 \\
\hline Target 9 & 0.596 & 0.417 & 0.382 & 0.077 \\
\hline
\end{tabular}

Entries in boldface $(\mathbf{0 . 8 9 1})$ are significant at $p<0.01$; those in italics $(0.077)$, not significant; others, significant at $p<0.05$.
The results shown in Figure 10 address one final question: how much is aclually saved by adopting the actual final arm posture? We show the manner in which the work done (according to Eq. 7) is predicted to vary as the amount of humeral rotation varies from $0^{\circ}$ (vertical plane of the arm) to $90^{\circ}$. Data are shown for two subjects ( 1 in A, 2 in B) and for three initial locations (indicated by line type) for two targets each. In each instance, the initial locations were chosen because they spanned the range of experimental and predicted values of humeral rotation. As shown in Figures 3 and 4, the plane of the arm varies most for target 5 and least for targets 7 and 9 . Each of the curves is normalized with respect to the minimum work possible for that combination of starting and final target locations. The data points on each of the curves correspond to the experimental values for final humeral rotation.

Table 3. Correlation coefficients $\left(r^{2}\right)$ between predicted and actual inclination $(v)$ of the plane of the arm

\begin{tabular}{lllll} 
& Subject 1 & Subject 2 & Subject 3 & Subject 4 \\
\hline Overal1 & $\mathbf{0 . 7 7 4}$ & $\mathbf{0 . 7 6 7}$ & $\mathbf{0 . 7 6 9}$ & $\mathbf{0 . 6 2 0}$ \\
Target 1 & $\mathbf{0 . 9 1 8}$ & $\mathbf{0 . 8 3 5}$ & $\mathbf{0 . 7 4 5}$ & $\mathbf{0 . 6 6 3}$ \\
Target 3 & $\mathbf{0 . 6 1 6}$ & $\mathbf{0 . 5 3 7}$ & $\mathbf{0 . 7 7 1}$ & 0.238 \\
Target 5 & $\mathbf{0 . 8 4 0}$ & $\mathbf{0 . 8 7 1}$ & $\mathbf{0 . 8 5 0}$ & 0.245 \\
Target 7 & $\mathbf{0 . 6 0 8}$ & $\mathbf{0 . 7 5 5}$ & $\mathbf{0 . 4 1 1}$ & $\mathbf{0 . 4 4 6}$ \\
Target 9 & $\mathbf{0 . 5 8 0}$ & $\mathbf{0 . 7 1 6}$ & $\mathbf{0 . 7 7 5}$ & 0.230 \\
\hline
\end{tabular}

Entries in boldface (0.774) are significant at $p<0.01$; others, at $p<0.05$. 


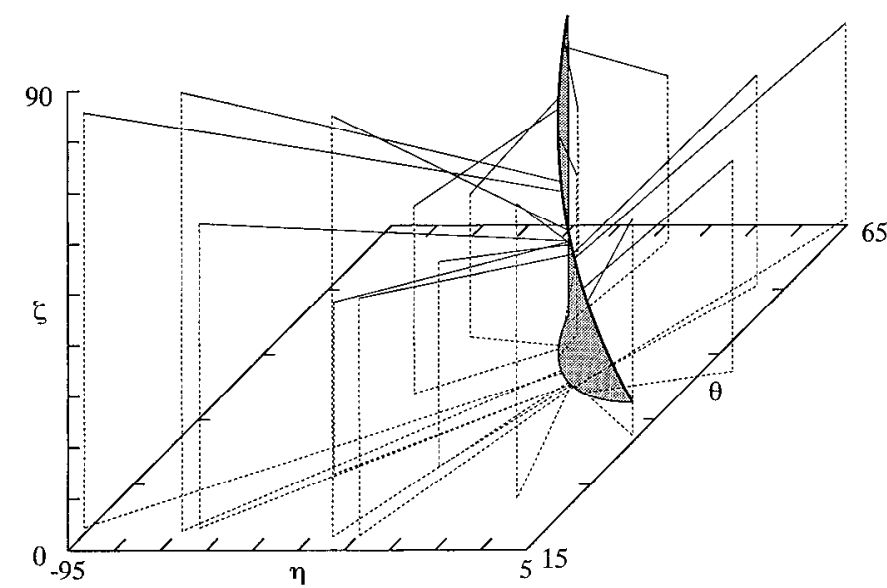

Figure 9. Distribution of predicted joint trajectories. The data are plotted in the same format as those in Figure 5, using the values of final arm posture predicted by minimizing peak work.

Typically, the amount of work required to move the arm into a nonoptimal posture is predicted to be as much as twice the work for the optimal solution. However, there are instances in which the amount of work required varies very little with the amount of humeral rotation at the target (for example, the dotted trace for Target 9, Fig. 10A) or varies as much as three times (dashed line, Target 9 in Fig. 10A). In all of the instances illustrated, the experimental data were within $5 \%$ of the optimal solution.

\section{Discussion}

In this article, we have concluded that Donders' law does not hold for pointing movements of the arm. Furthermore, we were able to predict the final posture of the arm under the assumption that the movement was performed as "effortlessly" as possible, that is, by expending the minimum amount of energy. In this Discussion, we will first attempt to reconcile our observations with those of previous investigations. 'Then we will discuss the simplifying assumptions made in our computations. Finally, we will attempt to relate our approach to previous approaches incorporating optimization techniques.

Relation to previous work. In some aspects, our present data agree with the results presented in previous investigations (Straumann et al., 1991; Hore et al., 1992; Miller et al., 1992), even though we come to a different interpretation. In our experimental conditions, torsion $(T)$ had a standard deviation that averaged $5.2^{\circ}$ about the mean value at each target location. This result is about twice the value $\left(2.5^{\circ}\right)$ reported by Hore et al. (1992). Straumann et al. (1991) reported that the Listing's plane for the arm had a thickness that ranged up to $2.7^{\circ}$. (Their measure is roughly comparable to the variation in torsion.) Miller et al. (1992) reported somewhat larger values, ranging from about $3^{\circ}$ to $6^{\circ}$. Thus, in this respect our present results do not differ substantially from previous observations. However, we also found that the torsional rotation $(T)$ of the arm could be as large as $50^{\circ}$. Hore et al. (1992), who examined a similar range of pointing directions $\left( \pm 45^{\circ}\right.$ in azimuth and elevation), found that torsion never exceeded $\pm 15^{\circ}$. Straumann et al. (1991), for a smaller range of pointing directions, also found the amount of torsion of the arm to be small.

As described in the introductory section, these previous investigations involved pointing to distant targets with an arm that
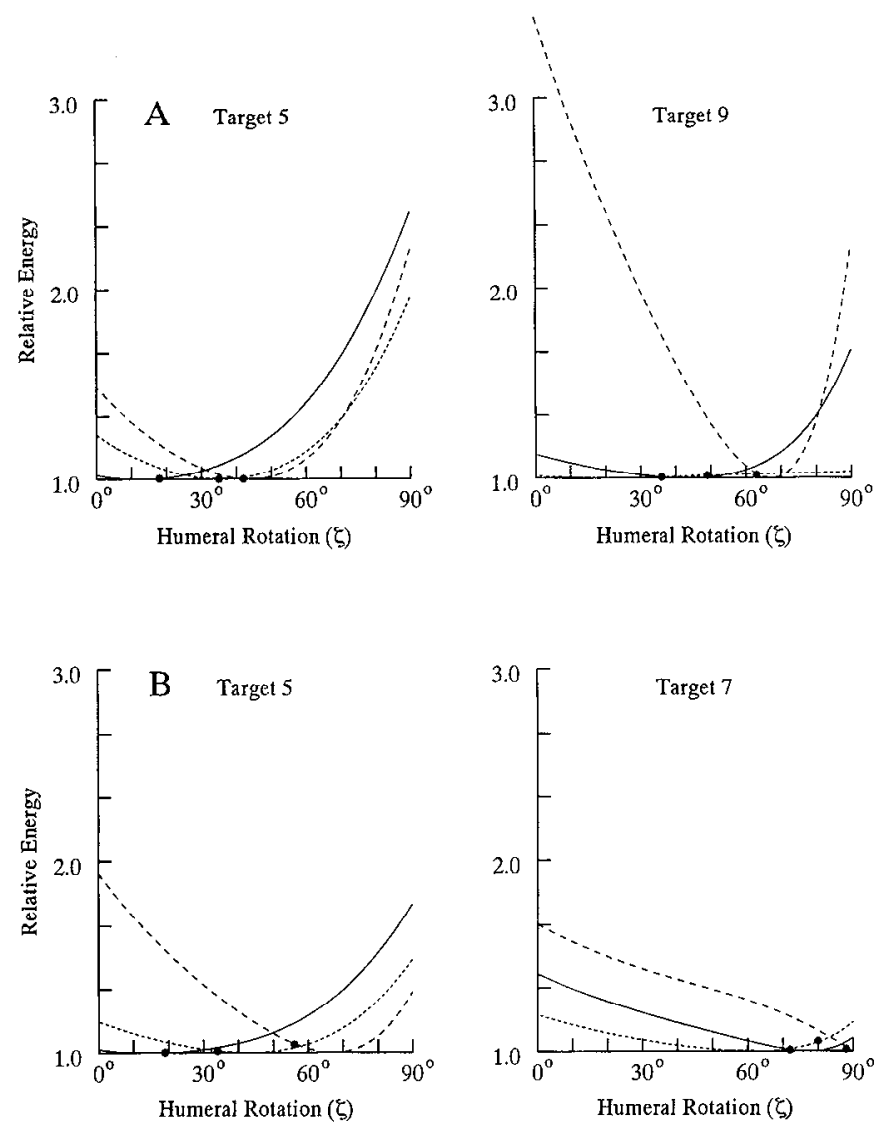

Figure 10. Relative energy requirements to move the arm into different postural configurations. Each trace denotes the relative amount of energy required to move the arm from a given initial hand location to the indicated target, plotted as a function of the amount of humeral rotation $(\zeta)$ at the target location. The data were normalized to the minimum value. The actual arm posture for each of the starting locations is indicated by the solid cirrles.

was either fully extended or close to fully extended. In our experiments, we also varied the distance of the target from the shoulder. The differing amounts of absolute torsion observed in the previous studies and in the present one indicate that there is no unique relationship between the direction of the target and the arm's torsional rotation; torsion also changes with the elbow angle, or equivalently, with the distance of the target from the shoulder.

In agreement with previous investigations, the inclination $v$ of the plane of the arm (or the torsional rotation of the arm) was found not to vary over its full biological range. Furthermore, at any one target location, the range of values observed did not exceed $30^{\circ}$ (Figs. 3,4 ). Nevertheless, the variability we observed was not random; the plane of the arm was found to depend significantly on the starting location of the hand as well as on the target's location. We propose that the variation is due (at least in part) to kinetic factors.

We have previously presented a model to account for the kincmatic sensorimotor transformations in pointing movements to visual targets (Flanders et al., 1992). In that model, we proposed that visually derived information about the location of the target was transformed, by means of linear approximations, into the arm angles $(\eta, \theta$, and similar measures for the forearm) that would put the hand on the target. According to the model, the posture of the arm, at the end of a pointing movement to a virtual 
target, depends only on the target's location, but not on the initial posture of the arm. Our present results suggest that this model is incomplete: initial arm posture must be taken into account as well. The previous model and our present observations lead to the following set of predictions. (1) Errors in pointing to virtual targets should depend on target location and initial hand location, and (2) for each initial hand location, there should be a linear mapping between target location and arm angles, but the coefficients of this linear mapping should depend on the initial posture of the arm. If these predictions are satisfied, the previous model would need to be revised only modestly. Alternatively, it is possible that some of the previous observations were a fortuitous happenstance of the initial arm posture that was chosen.

Simplifying assumptions. To estimate the amount of work required to propel the arm to the target, we made scveral simplifying assumptions. The first of these was that each of the angular velocities reached their maximum at the same time. It is known (Morasso, 1981) that the tangential velocity of the wrist is single peaked. In fact, the velocity profile of the wrist during pointing movements has commonly been characterized as being bellshaped (Hogan et al., 1987). However, this by itself is not sufficient to ensure that all of the components of angular velocity reach their maximum at the same time. Experimental observations (Soechting and Lacquaniti, 1981; Lacquaniti et al., 1986) suggest that this assumption is nevertheless a reasonable one.

The second assumption we made was that each of the peak angular velocities was proportional to the differences between the final and initial angles. This assumption will be correct if each of the angular velocities reaches a peak at the same time and if angle-angle plots are rectilinear. This latter condition is sometimes violated (cf. Soechting and Lacquaniti, 1981). In fact, Hollerbach and Atkeson (1986) have suggested that joint angular trajectories can be more accurately approximated by a staggered joint interpolation, the angle having the least excursion beginning to change later in the movement. At this point, it is difficult to predict to what extent a more accurate model of joint trajectories would have affected our results.

Taking actual joint trajectories into consideration would have required a considerably more sophisticated analysis. We would not only have needed to optimize the final posture, but to optimize among the infinite possible trajectories between the initial and final postures. Thus our prediction represents a first approximation to an optimal solution, a further refinement being provided by fine-tuning the actual path of the wrist and the trajectory of joint angles during the movement. It is conceivable that such an approach could in fact account for experimental variations in trajectories for movements performed in different directions (see the work of Uno et al., 1989, discussed below). For example it is known that for some pointing movements, the hand follows a path that is nearly straight, whereas the path exhibits considerable curvature for other target locations (Atkeson and Hollerbach, 1985; Hollerbach and Atkeson, 1986; Lacquaniti et al., 1986; Flanders et al., 1994). Furthermore, shoulder and elbow torques do not always attain their maximum at the same time (Buneo et al., 1995). The activation of individual shoulder and elbow muscles exhibits temporal shifts relative to each other, the temporal shifts being direction dependent (Flanders, 1991). It is tempting to speculate that an extension of the approach we developed here could account for these phenomena as well.

We made one additional assumption in our computations; we did not include the effect of gravity. The amount of torque that would need to be exerted to counteract the gravitational load of the arm will also depend on the inclination of the plane of the arm. If this load were to be minimized, one would expect a unique value of arm inclination $(v)$ for any particular target. This was clearly not the case in our experiments. However, it is possible that a combination of static factors (gravitational loads) and dynamic factors (work, as per Eq. 7) is minimized. If so, static factors would dominate for slow movements, whereas dynamic factors would be dominant for fast movements. Therefore, one would predict that the final posture of the arm would in general depend on the speed of the movement, for movements from any point a to any point $b$. We did not investigate this possibility in the present experiments. However, previous investigations (Soechting and Lacquaniti, 1981; Lacquaniti et al., 1986) have shown that arm trajectories are independent of the speed with which they are performed. Furthermore, both theoretical (Hollerbach, 1984) and experimental considerations (Flanders and Herrmann, 1992) suggest that static (gravity-dependent) and dynamic (speed-dependent) aspects of the movement are controlled separately and that both are generated from templates that are scaled appropriately in time and amplitude.

In summary, it is likely that a more sophisticated and detailed analysis would lead to predictions that are slightly different from those derived using the simplifying assumptions. It is unlikely, however, that the general trends would be altered. Because the moment of inertia of the arm about axes perpendicular to the humeral axis is 50-100 times as great as is the moment of inertia about the arm's long axis, rotations of the arm that maximize the amount of rotation about the humeral axis and minimize the amount of rotation about axes perpendicular to the humeral axis will be energetically efficient. The results in Figure 10 show that the saving can be substantial.

We should also point out that our analysis was able to predict the distribution in the inclination of the plane of the arm $(v$ Table 3 ) and the amount of humeral rotation ( $\zeta$, Table 2 and Fig. 8) across all five target locations, that is, the same model was valid for all five target locations. For each target location, the average experimental value of $\zeta$ coincided with the average predicted value. However, for some target locations, we found that $\zeta$ varied less than was predicted (e.g, target 7 in Fig. 8). Thus, there may be an additional constraint, acting to maintain a constant orientation of the arm, provided that the energetic cost of this constraint is not too high. This supposition may account for the results of previous investigations involving pointing to distant targets with an arm that was close to fully extended. With the arm extended, the energetic cost of changing arm posture by rotations about the long axis of the arm will be vanishingly small since the moment of inertia about this axis is only about $1 \%$ of the value of the moment of inertia about a perpendicular axis.

How does the present hypothesis relate to previous approaches? Other investigators have also approached the question of the control of multi-degree-of-freedom arm movements from the perspective that some parameter is optimized. Thus, Hogan and Flash (1987) have proposed that movements are performed as smoothly as possible. More, precisely, they suggested that jerk (the time derivative of acceleration) was minimized. At first glance, their approach and ours appear to he very similar, in that one might expect that movements that are performed smoothly would also be performed with minimum effort.

In fact, their hypothesis leads to predictions that are very different from ours. In particular, the "minimum jerk" hypothesis predicts that all hand paths should be rectilinear. Furthermore, since their hypothesis deals only with the trajectory of the hand, 
and not with the details of angular motion at the shoulder, their hypothesis makes no predictions concerning the orientation of the plane of the arm for different movements. Finally, "minimum jerk" at the hand does not ensure maximum smoothness of angular motion at the shoulder and elbow. Such a criterion, imposed at the joint level, leads to predicted hand paths that are very different from those predicted by Hogan and Flash's hypothesis as well as from those that are observed experimentally.

The approach taken by Uno et al. (1989) is closer to the one we have taken. They evaluated what they termed a "minimum torque change" model for arm movements restricted to two degrees of freedom (flexion/extension at the shoulder and elbow). They used the criterion that the integral of the sums of the squares of the time derivatives of torque be minimum to predict the hand trajectory. Their model was quite successful, predicting the curvature of handpaths for a variety of movements. Our approach is similar to theirs in that we suggest that what is optimized is closer to the dynamics of the movement than to its kinematics, but it is not clear if their criterion leads to the same predictions as does ours. We chose minimum work as a criterion because it was both more intuitive and easier to implement mathematically.

In this article we have tried to show that the hypothesis that the CNS attempts to minimize the expenditure of energy during movement can predict the posture of the arm at the end of a pointing movement. If our hypothesis is correct, it accounts for part of what the control mechanisms attempt to achieve during movement. In fact, we suspect that the CNS does not act to optimize a single parameter (such as peak work), but that the observed behavior results from a multitude of constraints.

\section{References}

Alpern M (1969) Kinematics of the eye. In: The eye (Davson H, ed), pp 13-25.

Atkeson CG, Hollerbach JM (1985) Kinematic features of unrestrained vertical arm movements. J Neurosci 5:2318-2330.

Buneo CA, Boline J, Soechting JF, Poppele RE (1995) On the form of the internal model for reaching. Exp Brain Res, in press.

Crawford JD, Vilis T (1991) Axes of eye rotation and Listing's law during rotations of the head. J Neurophysiol 65:407-423.

Flanders M (1991) Temporal patterns of muscle activation for arm movements in three-dimensional space. J Neurosci 11:2680-2693.

Flanders M, Herrmann U (1992) Two components of muscle activation: scaling with the speed of arm movement. J Neurophysiol 67: 931-943.

Flanders M, Helms Tillery SI, Soechting JF (1992) Early stages in a sensorimotor transformation. Behav Brain Sci 15:309-362.

Flanders M, Pellegrini JJ, Soechting JF (1994) Spatial/temporal characteristics of a motor pattern for reaching. $J$ Neurophysiol 71:811813.
Helms Tillery SI, Ebner TJ, Soechting JF (1995) Task dependence of primate arm postures. Exp Brain Res, in press.

Hogan N, Flash T (1987) Moving gracefully: quantitative theories of motor coordination. Trends Neurosci 10:170-174.

Hogan N, Bizzi E, Mussa-Ivaldi FA, Flash T (1987) Controlling multijoint motor behavior. Exerc Sports Sci Rev 15:153-190.

Hollerbach JM (1984) Dynamic scaling of manipulator trajectories. J Dyn Syst Meas Contr 106:102-106.

Hollerbach JM, Atkeson CG (1986) Characterization of joint interpolated arm movements. Exp Brain Res Ser 15:41-54.

Hore J, Watts S, Vilis T (1992) Constraints on arm position when pointing in three dimensions: Donders' law and the Fick gimbal strategy. J Neurophysiol 68:374-383.

Keshner EA, Baker JF, Banovetz J, Peterson BW (1992) Patterns of neck muscle activation in cats during reflex and voluntary head movements. Exp Brain Res 88:361-374.

Lacquaniti F, Soechting JF, Terzuolo CA (1986) Path constraints on point-to-point arm movements in three-dimensional space. Neuroscience 17:313-324.

Miller LE, Theeuwen M, Gielen CCAM (1992) The control of arm pointing movements in three dimensions. Exp Brain Res 90:415-426.

Morasso P (1981) Spatial control of arm movements. Exp Brain Res 42:223-237.

Nakayama K, Balliet R (1977) Listing's law, eye position sense and perception of the vertical. Vision Res 17:453-457.

Nelder JA, Mead R (1964) A simplex method for function minimization. Comp J 7:308--313.

Press WH, Teukolsky SA, Vetterling WT, Flannery BP (1992) Numerical recipes in C, Sccond cdition. Ncw York: Cambridge UP.

Robinson DA (1982) The use of matrices in analyzing the three-dimensional behavior of the vestibulo-ocular reffex. Biol Cybern 46: 53-66.

Simpson JI, Graf W (1985) The selection of reference frames by nature and its investigation. Rev Oculomot Res 1:3-20.

Soechting JF, Flanders M (1993) Parallel, interdependent channels for location and orientation in sensorimotor transformations for reaching and grasping. J Neurophysiol 70:1137-1150.

Soechting JF, Lacquaniti $F$ (1981) Invariant characteristics of a pointing movement in man. J Neurosci 1:710-720.

Soechting JF, Ross B (1984) Psychophysical determination of coordinate representation of human arm orientation. Neuroscience 13:595604.

Socchting JF, Lacquaniti F, Terzuolo CA (1986) Coordination of arm movements in three-dimensional space. Sensorimotor mapping during drawing movement. Neuroscience 17:295-311.

Straumann D, Haslwanter T, Hepp-Reymond M-C, Hepp K (1991) Listing's law for eye, head and arm movements and their synergistic control. Exp Brain Res 86:209-215

Tweed, D, Vilis, T (1987) Implications of rotational kinematics for the oculomotor system in three dimensions. J Neurophysiol 58:832-849.

Tweed D, Vilis T (1990) Geometric relations of eye position and velocity vectors during saccades. Vision Res 30:111-127.

Uno Y, Kawato M, Suzuki R (1989) Formation and control of optimal trajectory in human multijoint arm movement. Minimum torque change model. Biol Cybern 61:89-102.

Westheimer G (1957) Kinematics of the eye. J Optic Soc Am 47:967974. 